\title{
Peper Designing a Compact Microstrip Antenna Using the Machine Learning Approach
}

\author{
Kanhaiya Sharma and Ganga Prasad Pandey \\ School of Technology, Pandit Deendayal Petroleum University, Gandhinagar, India
}

https://doi.org/10.26636/jtit.2020.143520

\begin{abstract}
This paper presents how machine learning techniques may be applied in the process of designing a compact dual-band H-shaped rectangular microstrip antenna (RMSA) operating in $0.75-2.20 \mathrm{GHz}$ and $3.0-3.44 \mathrm{GHz}$ frequency ranges. In the design process, the same dimensions of upper and lower notches are incorporated, with the centered position right in the middle. Notch length and width are verified for investigating the antenna. An artificial neural network (ANN) model is developed from the simulated dataset, and is used for shape prediction. The same dataset is used to create a mathematical model as well. The predicted outcome is compared and it is determined that the model relying on ANN offers better results.
\end{abstract}

Keywords—artificial neural network, dual band, microstrip antenna, notch.

\section{Introduction}

New developments in the field of wireless communications call for compact, wide-band, high efficiency, multiband, and low cost antennas that are suitable for modern day applications. Microstrip antennas offer numerous advantages, such as small size, low weight and ease of fabrication due to their planar configuration. An antenna of this type is easy to mount on a rigid surface, supports dual-polarization and, unlike solutions of other types, multiband operation. The different techniques relied upon to make the antenna compact [1] without affecting its basic parameters include reactive loading [2], using a monopole antenna with defected ground structure (DGS), and modified patch [3]-[7]. Those methods are capable of reducing the antenna size by up to $70 \%$. Meta-material [8]-[14] and engineered ground structure [15]-[18] may reduce the size of the antenna by up to $74 \%$ as well. Other miniaturization methods, like deployment of fractal structures in antenna design [19]-[21] may reduce the size of the antenna by up to $75 \%$ and engineered substrates [22]-[24] allow to reduce the size of antenna by $80 \%$ and by introducing close-ended or openended slots [25]-[30] of various shapes H, L, U, E, V, W, $\mathrm{D}, \mathrm{S}$ double $\mathrm{E}$, double $\mathrm{U}$ may reduce the size of the antenna by up to $86.5 \%$. In some cases, more than one technique is used to make the antenna as compact as possible.

Machine learning (ML) is a subset of artificial intelligence (AI), effectively used in all areas of engineering, espe- cially in communication network automation. ML is a specific type of data analytic techniques enabling machines to learn from experience (training) and to predict new data. A comparison between the traditional and the ML approach is given in Fig. 1. ML is preferred to solve complex mathematical problems with lots of variables and massive amounts of data. Today's methods relied upon while designing antennas require such a high level of expertise, as in most cases the design process requires a trade-off between such antenna parameters as small size and good bandwidth. AI approaches have been used in antenna design and optimization since the 1990s [31]. The most significant advantages that the use of the AI approach in antenna design has over the traditional methods lies in the ability to simultaneously handle multi-objective problems in order to achieve the specified goal and to provide the best automated solution [32]-[35].

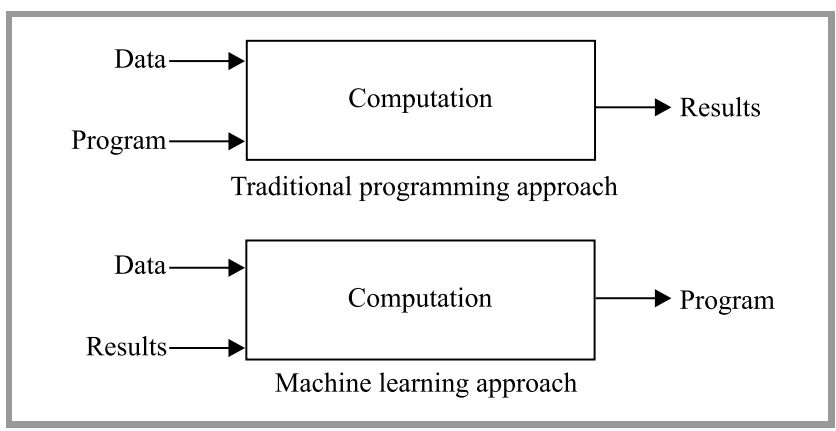

Fig. 1. Computational approaches.

The drawback of analytical-based methods consists in bigcomputation overheads experienced in the design process. To cope with this, the trial-and-error approach may be used to achieve the specified goals. Slot-loading turns out to be the technique that is most widely used among all aforementioned methods allowing to make antennas compact [28] in size.

\section{Synthesis and Analysis Problem for Microstrip Patch Antenna Using ANN}

ANN is one of the approaches used in ML to map nonlinear data efficiently [36], [37] based on experience (training), 


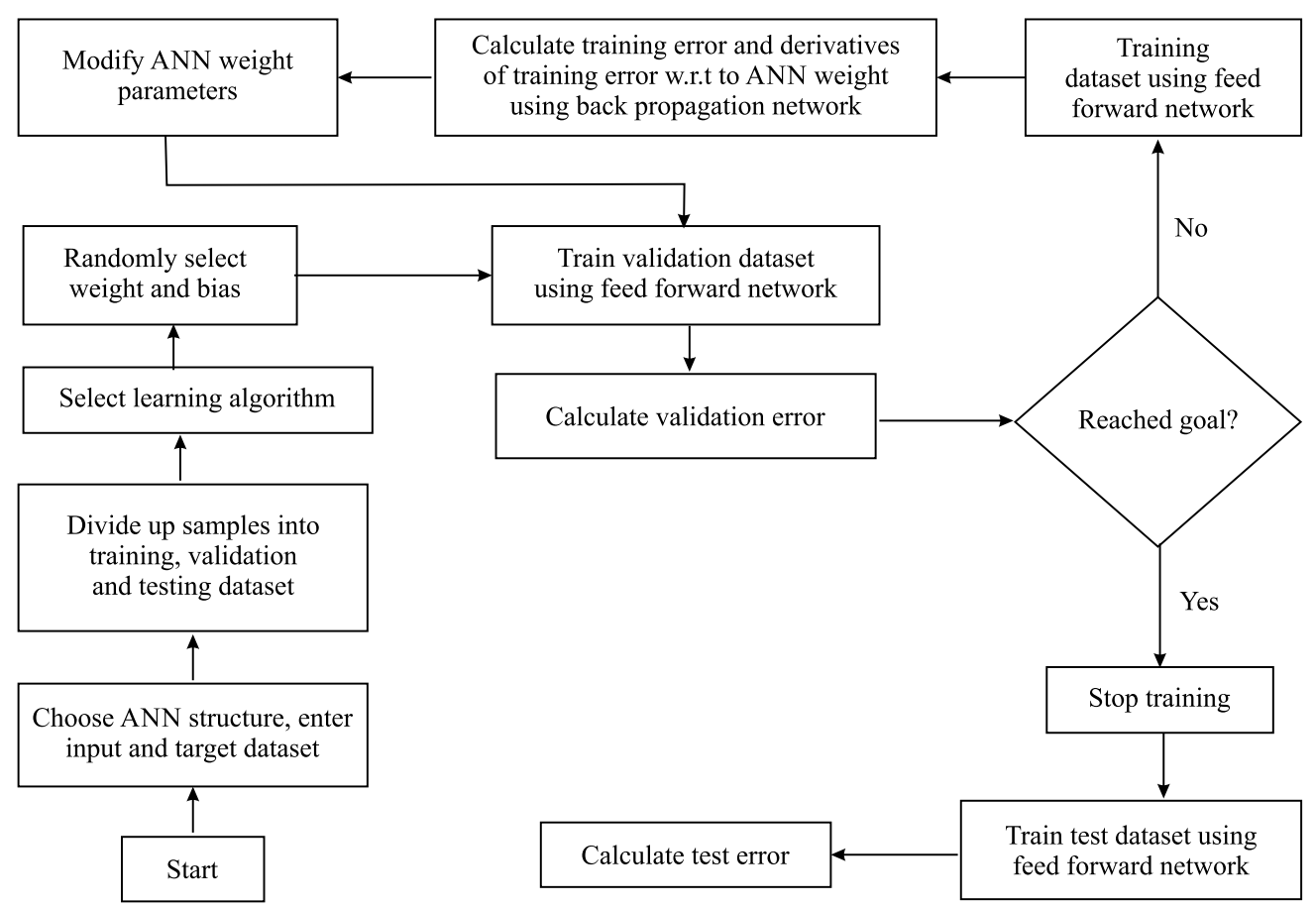

Fig. 2. Flowchart of ANN back-propagation algorithm.

while dealing with new data. Basically, three logic layers are distinguished in ANN architectures. The first is known as the input layer, while the last is referred to as the output layer. Between the input and the output layer, there is a number of hidden layers. Each hidden layer has one or more neurons. The number of the hidden layers, neurons, the activation function and the learning algorithm are all specific to the application. There are no rules to determine the number of hidden layers and neurons, but in most problems two to three hidden layers are used at the most in order to approximate all types of mathematical functions. The performance of ANN model depends upon the data collection, learning algorithm, weight initialization, change in an activation function, etc. In the case of antenna design, data should be collected either through simulations or measurements. The range of data always extends, marginally, beyond the model's utilization range [38]. Initially, data samples are divided into three sets, known as training (with the usage ratio of $70 \%$ ), testing (15\%), and validation $(15 \%)$. According to the needs of a specific application, the percentages may be different. Next, the network size is chosen, i.e. the number of hidden layers and neurons in each of them is determined. Finally, the algorithm is selected based on feed-forward, back propagation, and feed-forward back propagation, to train the ANN and obtain the model. A flowchart of ANN feed-forward back-propagation algorithms is presented in Fig. 2 .

To achieve the minimum mean squared error (MSE) It is necessary to train the model with accurate data. In this case, a total of 80 samples has been collected by simulating $\mathrm{H}$-shaped RMSA using IE3D software. To minimize
MSE, different ANN algorithms have been tested by varying the number of hidden layers and neurons in each hidden layer. Finally, the best combination for the proposed model was selected. To train the ANN model, highly accu-

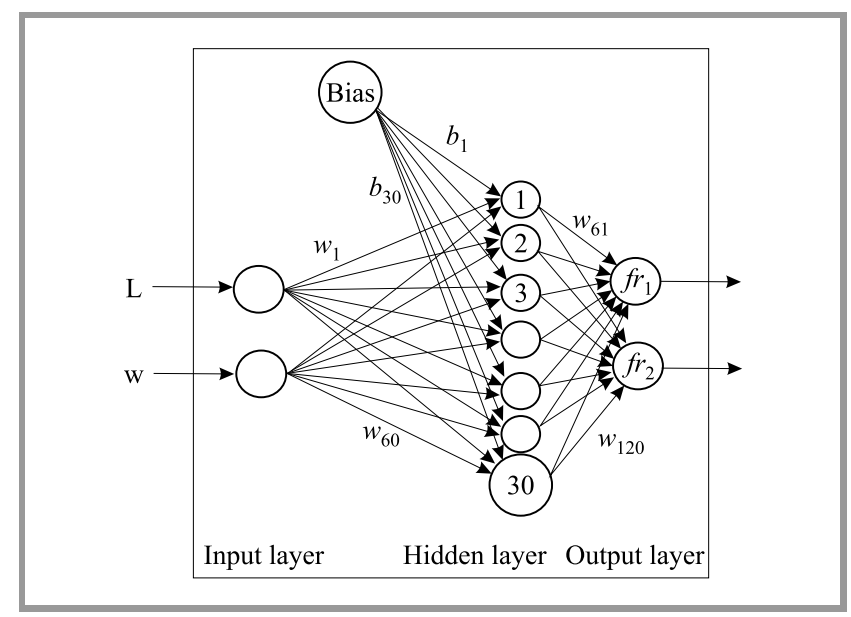

Fig. 3. Proposed ANN model for the analysis of compact H-shaped RMSA.

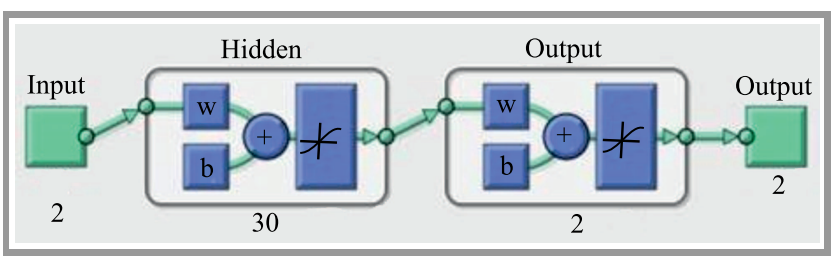

Fig. 4. ANN network architecture to train physical-to-electrical parameter. 

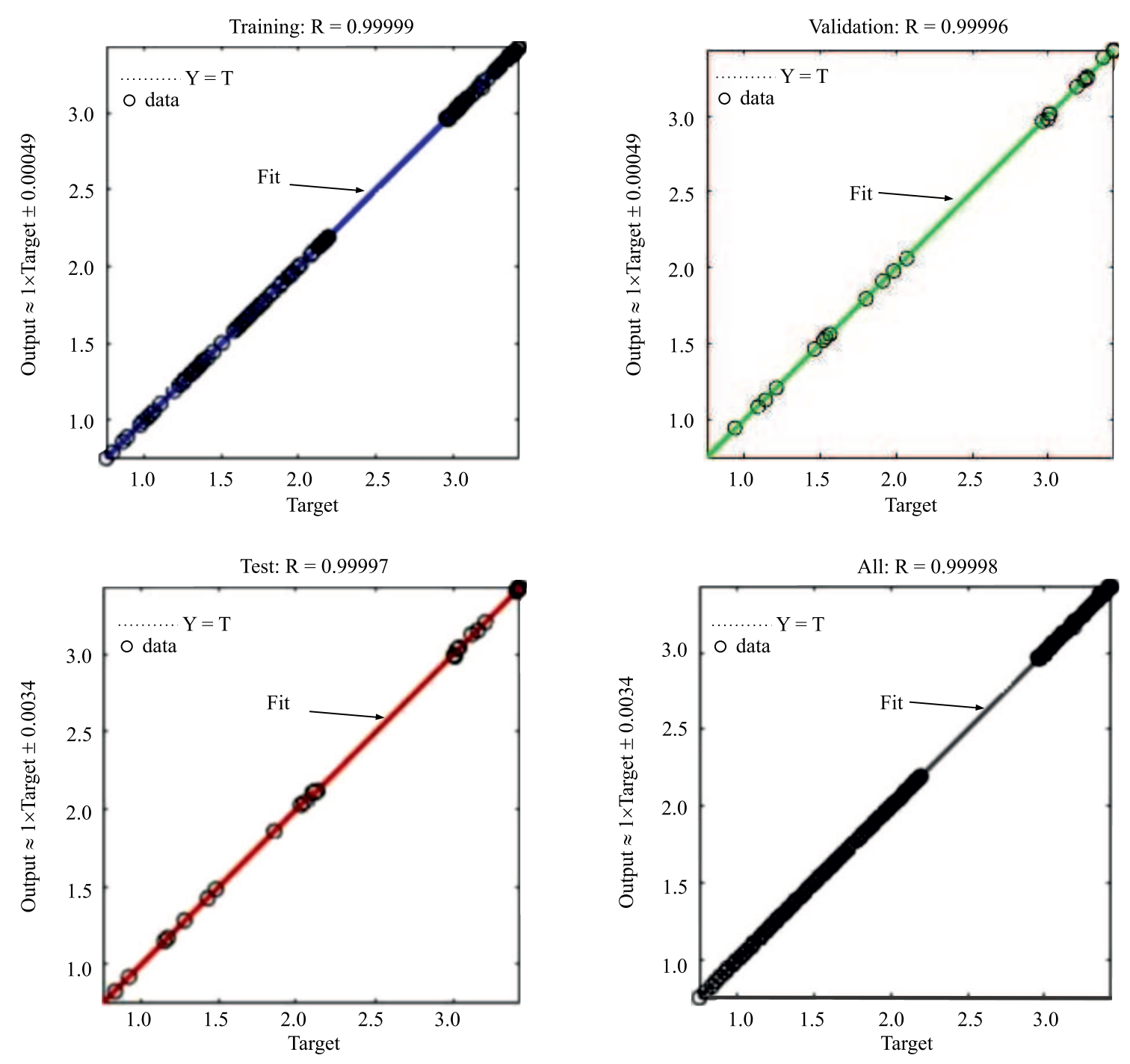

Fig. 5. Regression plot for physical to electrical parameters.

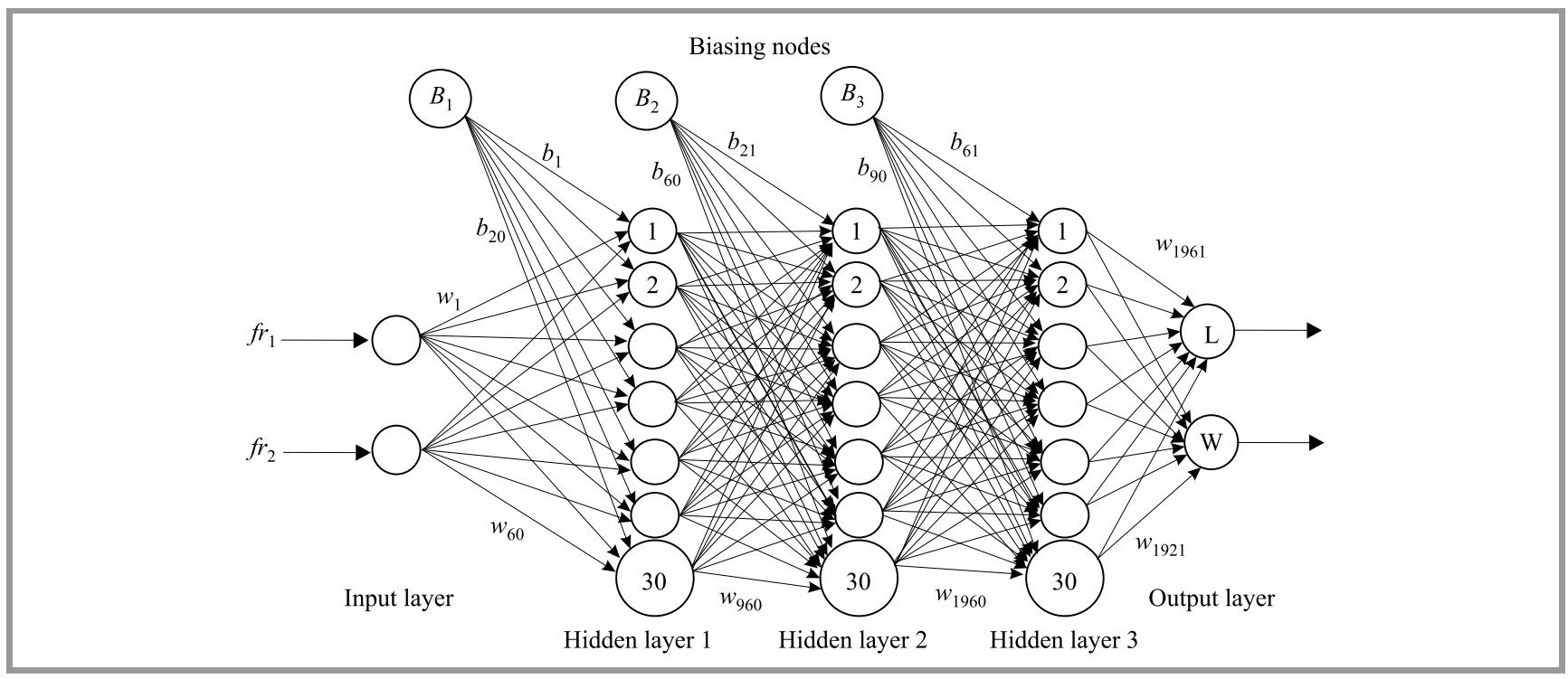

Fig. 6. Proposed ANN model for synthesis of H-shaped compact RMSA. 
rate back propagation algorithms with minimum MSE were used [39].

Figure 3 shows a detailed architecture of the hidden layer of the proposed ANN model which uses the train set and predicts the dual resonant frequencies $\left(f_{r 1}, f_{r 2}\right)$, as well as the physical aerial parameters, i.e. slot length (L) and width (W) as input values. The Levenberg-Marquardt (LM) back propagation algorithm is used as ANN. ANN network architecture is shown in Fig. 4 with two input and output parameters, one hidden layer and 30 neurons. The regression plot of training, testing, validation, and overall process is shown in Fig. 5.

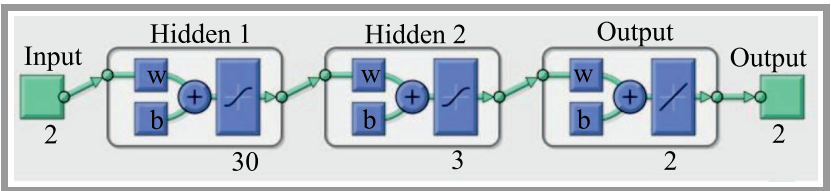

Fig. 7. ANN network architecture to train electrical-to-physical parameters.

To train the ANN model for predicting physical dimensions of antenna, Bayesian Regularization (BR) back propagation algorithm have been used. ANN network architecture is shown in Figs. 6-7, where input and output parameters equals to two, the number of hidden layer is three, each hidden layer consists of thirty neurons. the regression plot of training, testing, and overall process is shown in Fig. 8. In the next step, using the same dataset, a mathematical model was developed to map the dominant resonance mode $\left(T M_{10}\right)$ and the higher mode $\left(T M_{02}\right)$ of a dual band compact $\mathrm{H}$-shaped patch antenna using the curve fitting tool in Matlab software. Equation 1 represents the dominant mode $\left(T M_{10}\right)$ and Eq. 2 represents the higher mode $\left(T M_{02}\right)$ :

$$
\begin{gathered}
f r_{1}=2.279-0.145 l+.07311 w \\
f r_{2}=3.37+0.1202 l-0.081 w-0.05906 l^{2}+ \\
0.04877 l w+0.002621 l^{3}-0.002256 l^{2} w .
\end{gathered}
$$

\section{Result and Discussion}

Analysis of the H-shaped compact RMSA using the proposed ANN model is performed by taking one hidden layer and 30 neurons, as shown in Fig. 4. 80 samples were taken to train the model, with $70 \%$ used for training, $15 \%$ for validation and $15 \%$ for testing. Figure 5 shows the regression plots for training, validation, testing, and the overall process. The regression $\mathrm{R}$ value indicating the correlation between output and target, $\mathrm{R}$ value closer to one means that the close relationship. In the ideal case, both outputs and target are equal. It is clear from the evidence shown that the $\mathrm{R}$ value for training, validation, and testing of the proposed model is $99.998 \%$ accurate, meaning that it is perfect regression model. The synthesis of H-shaped compact RMSA using the proposed ANN model is performed by taking three hidden layers and 30 neurons per hidden layer, as shown in ANN network architecture in Figs. 6-7.
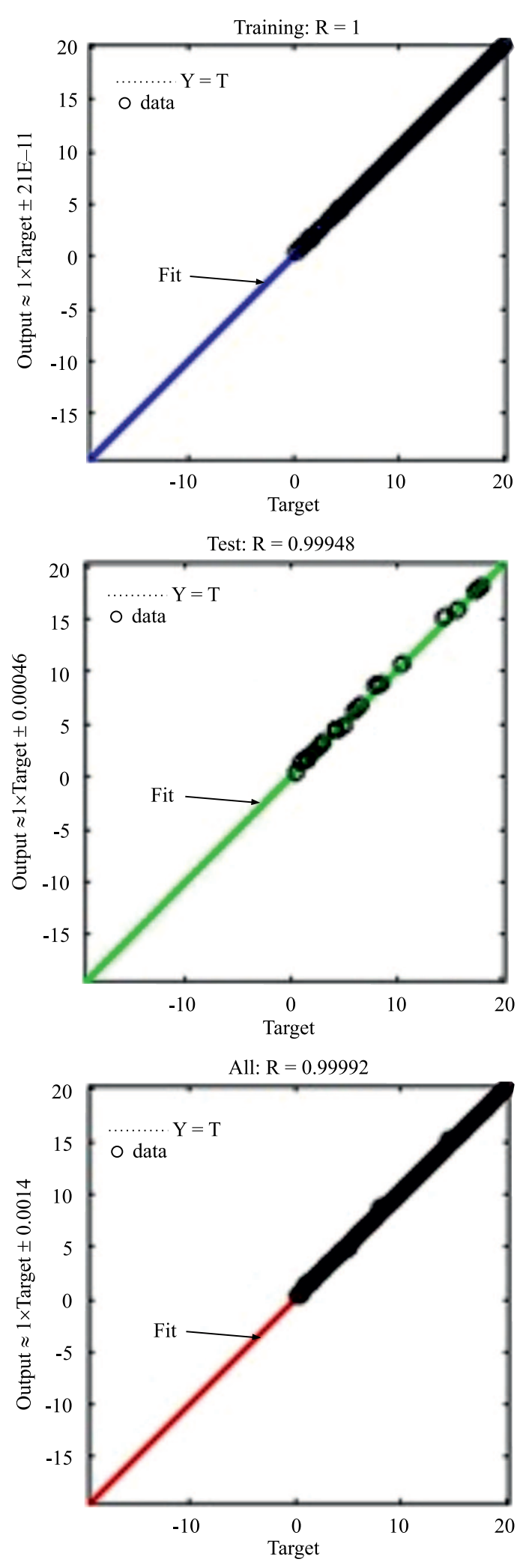

Fig. 8. Regression plot for electrical to a physical parameters.

In this case, 80 samples are used to train the model $(85 \%$ for training, $15 \%$ for testing). Figure 8 shows regression plots for training, testing, and the overall process. It is clear from the evidence shown that the regression $\mathrm{R}$ value 


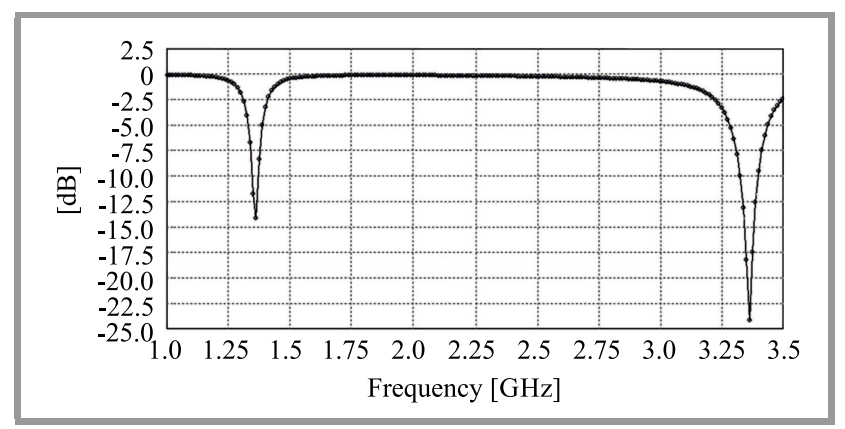

Fig. 9. $S_{11}$ vs. frequency plot for H-shaped RMSA antenna.

for training and testing of the proposed model is $99.992 \%$, which is very close to benchmark value 1 . In the ideal scenario, both output and target are equal. In both the cases, the variations are placed precisely on dotted line, as shown in Figs. 5 and 8.

Table 1 shows the result of the proposed ANN model used for analyzing a compact H-shaped RMSA. Except for a few samples, MSE is of the order of $10^{-5}$, which indicates that the predicted resonant frequencies are close to simulated results $\left(f_{r 1}, f_{r 2}\right)$. Table 2 shows that the MSE is of the order of $10^{-12}$, which is very close to actual physical slot dimensions. Table 3 shows a comparison of $f_{r 1}, f_{r 2}$ collected from simulation, curve fitting, and ANN prediction. The dual resonant frequencies predicted by the proposed ANN model are very close to the simulated ones. The relation between rectangular notch dimensions and resonant frequencies $f_{r 1}$ and $f_{r 2}$ for the dominant mode ( $T M_{10}$ and for the higher mode $T M_{02}$ ) are shown in Fig. 10. The dimension of notches is increased from $0.25 \times 0.5 \mathrm{~mm}$ to $20.00 \times 20.25 \mathrm{~mm}$, in $0.25 \mathrm{~mm}$ steps. $f_{r 1}$ started falling from 2.20 to $0.75 \mathrm{GHz}$, while $f_{r 2}$ first increased from $3.38 \mathrm{GHz}$ to $3.434 \mathrm{GHz}$, and then suddenly decreased down to $2.98 \mathrm{GHz}$, and started increasing again from 2.98 to $3.19 \mathrm{GHz}$.

To validate the performance of $\mathrm{H}$-shaped dual-band RMSA, one sample was chosen randomly with the same dimensions as those shown in Table 1 , with the notch size of $8 \times 14 \mathrm{~mm}$, as well as with identical lower and upper notches centered in the middle. The infinite substrate and the infinite ground plane were assumed and simulated using IE3D software. The performance of the antenna measured in terms of scattering parameter, gain and radiation pattern was analyzed. $S_{11}$ vs. frequency characteristics shown in Fig. 9, indicate that the antenna radiates at $1.360 \mathrm{GHz}$ and $3.366 \mathrm{GHz}$ for two modes $\left(T M_{10}\right.$ and $T M_{02}$ ). From Fig. 11, one may conclude that the gain of the antenna in question measured at $1.360 \mathrm{GHz}$ is $7.572 \mathrm{dBi}$, and equals, at the upper resonant frequency of $3.366 \mathrm{GHz}, 1.00 \mathrm{dBi}$. E-plane and $\mathrm{H}$-plane radiation patterns at $1.360 \mathrm{GHz}$ and $3.366 \mathrm{GHz}$ with infinite substrate and infinite ground plane dimensions (Fig. 12) show $T M_{10}$ and $T M_{02}$ modes of operation, which is quite normal for any MSA. The proposed aerial offers better results in terms of accuracy and time complexity, with a higher
Table 1

Physical-to-electrical parameters

\begin{tabular}{|c|c|c|c|c|c|c|c|}
\hline \multicolumn{8}{|c|}{$\begin{array}{l}\text { Prediction of physical parameters (slot length, slot width) } \\
\text { to electrical }\left(f_{r 1}, f_{r^{2}}\right) \text { data }\end{array}$} \\
\hline \multicolumn{2}{|c|}{ Slot $(l, w)$} & \multicolumn{2}{|c|}{ Simulation } & \multicolumn{2}{|c|}{ ANN prediction } & ANN $\mathrm{mc}$ & del error \\
\hline$L$ & & $f_{r 1}$ & $f_{r 2}$ & $f_{r 1}$ & $f_{r 2}$ & $f_{r 1}$ & $f_{r 2}$ \\
\hline$\overline{0.25}$ & $\overline{0.5}$ & $\overline{2.19209}$ & 3.38418 & 2.19419 & 3.37800 & $\overline{\overline{10 \mathrm{E}-03}}$ & $\overline{8 \mathrm{E}-1}$ \\
\hline 0.5 & 0.75 & 2.19128 & 3.38579 & 2.19032 & 3.37734 & $9.58 \mathrm{E}-04$ & $8.45 \mathrm{E}-03$ \\
\hline 0.75 & 1 & 2.18725 & 3.36158 & & & & $154 \mathrm{~F}-02$ \\
\hline 1 & 1.25 & & 3.36965 & & 3.37718 & & \\
\hline 1.25 & 1.5 & 2.17918 & 3.36965 & 2.17556 & 3.37798 & $3.62 \mathrm{E}-03$ & $-8.33 \mathrm{E}-03$ \\
\hline 1.5 & 1.75 & 2.17111 & 3.38579 & & 3.37965 & & $6.14 \mathrm{E}-03$ \\
\hline 1.75 & 2 & & 3.38 & & 3.38238 & & \\
\hline 2 & 2.25 & 2.15819 & 3.38983 & 2.15618 & 3.38633 & 2.0 & $3.50 \mathrm{E}-03$ \\
\hline 2.25 & 2.5 & 2.14689 & 3.38983 & 2.14848 & 3.39150 & & $-1.67 \mathrm{E}-03$ \\
\hline 2.5 & 2.75 & & 3.3 & & & & \\
\hline 2.75 & 3 & 2.1 & 3.40194 & 2.13 & 3.40449 & & $5 \mathrm{E}-03$ \\
\hline 3 & 3.25 & & 3.41001 & 2.11 & 3.41125 & & $-1.24 \mathrm{E}-03$ \\
\hline 3.25 & 3.5 & & 3.41 & & 3.4 & & \\
\hline 3.5 & 3.75 & 2. & 3.42373 & & 3.42232 & & $1 \mathrm{E}-03$ \\
\hline 3.75 & 4 & & 3.42938 & & & & \\
\hline 4 & 4.25 & & 3.42 & & & & \\
\hline 4.25 & 4.5 & & 3.42147 & & 3.42924 & & $-7.77 \mathrm{E}-03$ \\
\hline 4.5 & 4.75 & & 3.434 & & & & $\mathrm{E}-03$ \\
\hline 4.75 & 5 & & 3.42 & & 3.42 & & \\
\hline 5 & 5.25 & 1.99 & 3.42373 & 1.9 & 3.42897 & & $24 \mathrm{E}-03$ \\
\hline 5.25 & 5.5 & & 3,43 & & & & $\mathrm{E}-03$ \\
\hline 5.5 & 5.75 & & 3.42 & & 3.42 & & \\
\hline 5.75 & 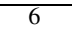 & 1.94 & 3.43019 & & 3.42941 & & $2 \mathrm{E}-04$ \\
\hline 6 & 6.25 & & 3.42 & & & & \\
\hline 6.25 & 6.5 & & 3.43 & & & & \\
\hline 6.5 & 6.75 & & 3.426 & & 3.42 & & $2 \mathrm{E}-03$ \\
\hline 6.75 & 7 & & 3.422 & & 3.42 & & $1 \mathrm{E}-03$ \\
\hline 7 & 7.25 & & 3.41 & & 3.41 & & $8 F=03$ \\
\hline 7.25 & 7.5 & & 3.406 & & 3.39 & & $9 \mathrm{E}-03$ \\
\hline 7.5 & 7.75 & & 3.40 & & & & $\mathrm{E}-02$ \\
\hline 7.75 & 8 & & 3.36 & & & & $\mathrm{~F}-03$ \\
\hline 8 & 8.25 & & 3.36 & & 3.3 & & $\mathrm{E}-03$ \\
\hline 8.25 & 8.5 & & 3.36 & & & & \\
\hline 8.5 & 8.75 & & 3.35 & & & & \\
\hline 8.75 & 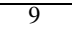 & & 3.344 & & 3.3 & & $\mathrm{E}-04$ \\
\hline 9 & 9.25 & & & & & & \\
\hline 9.25 & 9.5 & & 3.32 & & & & \\
\hline 9.5 & 9.75 & & 3.32 & & & & $\mathrm{E}-03$ \\
\hline 9.75 & 10 & & & & & & E-03 \\
\hline 10 & 10.25 & & & & & & \\
\hline 10.25 & 10.5 & & 3.28 & & & & -1 \\
\hline 10.5 & 10.75 & & & & & & \\
\hline 10.75 & 11 & & & & & & \\
\hline 11 & 11.25 & & 3.25 & & & & 20 \\
\hline 11.25 & 11.5 & & & & & & \\
\hline 11.5 & 11.75 & & & & & & \\
\hline 11.75 & 12 & & & & & & \\
\hline 10 & 12.25 & & & & & & \\
\hline 12. & 12.5 & & & & & & \\
\hline 12 & 12.75 & & & & & & \\
\hline 12.75 & 13 & & & & & & \\
\hline 13 & 13.25 & & & & & & \\
\hline 13.25 & 13. & & & & & & \\
\hline 13 & 13. & & & & & & \\
\hline 13.75 & 14 & & & & & & \\
\hline & 14.25 & & & & & & \\
\hline 14.25 & 14 & & & & & & \\
\hline 14 & 14.75 & & & & & & \\
\hline 14.75 & & & & & & & \\
\hline 15 & 15.2 & & & & & & \\
\hline 15.25 & 15. & & & & & & \\
\hline 15 & 15. & & & & & & \\
\hline 15.75 & $T$ & & & & & & \\
\hline 16 & 16.25 & & & & & & \\
\hline 62 & 16.5 & & & & & & \\
\hline 16 & 16.75 & & & & & & \\
\hline 16.75 & 17 & & & & & & \\
\hline 17 & 17.25 & & & & & & \\
\hline 725 & & & & & & & \\
\hline 17 & 17.75 & & & & & & \\
\hline 1775 & 18 & & & & & & \\
\hline 18 & 18.2 & & & & & & \\
\hline 18.25 & 18 & & & & & & \\
\hline 18 & 18.7 & & & & & & \\
\hline 18.75 & & & & & & & \\
\hline & 19.2 & & & & & & \\
\hline 19.2 & 19. & & & & & & \\
\hline 19 & 19.75 & & & & & & \\
\hline 19.75 & 20 & 79 & 3.18 & 0.7 & 3.16 & 2.9 & 2.2 \\
\hline & & & & & & & \\
\hline
\end{tabular}


Table 2

Electrical-to-physical parameters

\begin{tabular}{|c|c|c|c|c|c|c|c|}
\hline \multicolumn{8}{|c|}{$\begin{array}{l}\text { Prediction of electrical parameters }\left(f_{r 1}, f_{r 2}\right) \\
\text { to physical parameters (slot length, slot width) }\end{array}$} \\
\hline \multicolumn{2}{|c|}{ Slot $(l, w)$} & \multicolumn{2}{|c|}{ Input to ANN } & \multicolumn{2}{|c|}{ ANN prediction } & Error in pr & tion) \\
\hline$I_{-}$ & & & 12 & & & $f_{r 1}$ & $f_{r 2}$ \\
\hline$\overline{0.25}$ & $\overline{0.5}$ & 2.19209 & $\overline{38418}$ & $\overline{0.25000}$ & $\overline{.50000}$ & $\overline{4.26 \mathrm{E}-11}$ & $\overline{4.26 \mathrm{E}-}$ \\
\hline 0.5 & 0.75 & 2.19128 & 3.38579 & \begin{tabular}{|l|}
0.23763 \\
\end{tabular} & \begin{tabular}{|l|}
0.48763 \\
\end{tabular} & 0.26237 & \\
\hline 0.75 & 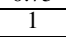 & \begin{tabular}{|l|}
2.18725 \\
\end{tabular} & 3.36158 & \begin{tabular}{|l|}
0.75000 \\
\end{tabular} & 1.00000 & $7.43 \mathrm{E}-11$ & $7.43 \mathrm{E}-11$ \\
\hline 1 & 1.25 & & & & & -0.25 & \\
\hline 1.25 & 4.4 & 2.17918 & 65 & 00 & 50000 & $.21 \mathrm{E}-11$ & $.21 \mathrm{E}-$ \\
\hline 1.5 & 1.75 & 2.17111 & 3.38579 & 1.50000 & 1.75000 & $5.05 \mathrm{E}-11$ & $5.05 \mathrm{E}-11$ \\
\hline 1.75 & 2 & & & & & & \\
\hline 2 & 2.25 & \begin{tabular}{|l|}
2.15819 \\
\end{tabular} & 3.38983 & \begin{tabular}{|l|}
2.05693 \\
\end{tabular} & 93 & 0.05693 & 5693 \\
\hline 2.25 & 2.5 & \begin{tabular}{|l|}
2.14689 \\
\end{tabular} & 3.38983 & & & $5.40 \mathrm{E}-11$ & \\
\hline 2.5 & 2.75 & & & & & & \\
\hline 2.75 & $\sqrt{3}$ & 1307 & 3.40194 & \begin{tabular}{|l|}
2.71920 \\
\end{tabular} & & .03080 & \\
\hline 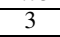 & 3.25 & 2.12268 & 3.41001 & & & -0.11056 & \\
\hline 3.25 & 3.5 & & & & & & \\
\hline 3.5 & 3.75 & 99040 & 3.42373 & 00 & & $58 \mathrm{E}-11$ & \\
\hline 3.75 & 4 & \begin{tabular}{|l|}
2.07910 \\
\end{tabular} & 3.42938 & & & $3.03 \mathrm{E}-11$ & \\
\hline 4 & 4.25 & & & & & & \\
\hline 4.25 & 4.5 & & 3.42147 & & & $95 \mathrm{E}-11$ & 11 \\
\hline 4.5 & 4.75 & 02986 & & & & 11 & \\
\hline 4.75 & & & & & & & \\
\hline 5 & 5.25 & 092 & 3.42373 & & & 70 & \\
\hline 5.25 & 5.5 & 1.98144 & & & & & \\
\hline 5.5 & 5.75 & 15 & & & & & \\
\hline 5.75 & 6 & 94915 & 3.43019 & & & $59 \mathrm{E}-12$ & \\
\hline 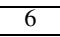 & 6.25 & 92655 & & & & & \\
\hline 6.25 & 6.5 & 0960 & & & & & \\
\hline 6.5 & 6.75 & 89266 & 3.42 & & & 09 & \\
\hline 6.75 & 7 & 88055 & & & & 11 & \\
\hline 7 & 7.25 & 6 & & & & & \\
\hline 7.25 & 7.5 & 1.83616 & 3.40 & & & 11 & \\
\hline 7.5 & 7.75 & 1.81356 & 3.40 & & & & \\
\hline 7.75 & 8 & 79984 & & & & & \\
\hline 8 & 8.25 & 77966 & & & & -0.5 & \\
\hline 8.25 & 8.5 & & & & & & \\
\hline 8.5 & 8.75 & 1 & & & & & \\
\hline 8.75 & re & 71 & 3.34 & & & -12 & \\
\hline & 9.25 & . & & & & & \\
\hline .25 & 9.5 & & & & & & \\
\hline 9.5 & 9.75 & & & & & -11 & \\
\hline 9.75 & 10 & & & & & & \\
\hline 10 & 10.25 & & & & & & \\
\hline 0.25 & 10.5 & & & & & & \\
\hline 10.5 & 10.75 & & & & & & \\
\hline 10.75 & 11 & & & & & & \\
\hline 11 & 11.25 & & & & & & \\
\hline 1.25 & 11.5 & & & & & & \\
\hline 11.5 & 11.75 & & & & & & \\
\hline 11.75 & 12 & & & & & & \\
\hline 12 & 12.2 & & & & & & \\
\hline 125 & & & & & & & \\
\hline 12.5 & 12. & & & & & & \\
\hline & 13 & & & & & & \\
\hline 13 & 13. & & & & & & \\
\hline 325 & 13 & & & & & & \\
\hline & 13 & & & & & & \\
\hline .75 & 14 & & & & & & \\
\hline 14 & 14.25 & & & & & & \\
\hline 425 & & & & & & & \\
\hline & 14. & & & & & & \\
\hline 14.75 & 12 & & & & & & \\
\hline & & & & & & & \\
\hline & & & & & & & \\
\hline & 15 & & & & & & \\
\hline & & & & & & & \\
\hline 10 & & & & & & & \\
\hline 16.2 & & & & & & & \\
\hline & & & & & & & \\
\hline 16.15 & & & & & & & \\
\hline 17 & & & & & & & \\
\hline 178 & & & & & & & \\
\hline 17. & 17.75 & & & & & & \\
\hline 17.75 & 18 & & & & & & \\
\hline 18 & & & & & & & \\
\hline 18. & 10 & & & & & & \\
\hline 18. & 18.7. & & & & & & \\
\hline & 19 & & & & & & \\
\hline 19 & 19.25 & & & & & -4. & \\
\hline 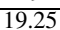 & 19. & & & & & & \\
\hline & 10 & & & & & & \\
\hline 19.7 & 20 & & & & & & \\
\hline & & & & & & & \\
\hline
\end{tabular}

Table 3

Comparison of resonant frequencies

Comparison of resonant frequencies in various methods \begin{tabular}{|c|c|c|c|c|c|c|c|}
\hline \multicolumn{7}{|c|}{ Slot size } & \multicolumn{2}{|c|}{ Simulation } & \multicolumn{2}{|c|}{ Curve fitting } & \multicolumn{2}{c|}{ ANN model } \\
\hline$L$ & $W$ & $f_{r 1}$ & $f_{r 2}$ & $f_{r 1}$ & $f_{r 2}$ & $f_{r 1}$ & $f_{r 2}$ \\
\hline
\end{tabular} \begin{tabular}{|c|c|c|c|c|c|c|c|}
\hline \hline 0.25 & 0.5 & 2.19209 & 3.38418 & 2.2793 & 3.3619 & 2.19323512 & 3.383955179 \\
\hline 0.5 & 0.75 & 2.19128 & 3.38579 & 2.2613 & 3.3728 & 2.190414971 & 3.377723607 \\
\hline 0.75 & 1 & 2.18725 & 3.36158 & 2.2434 & 3.3823 & 2.186759462 & 3.373556577 \\
\hline
\end{tabular} \begin{tabular}{|c|c|c|c|c|c|c|c|}
\hline 0.75 & 1 & 2.18725 & 3.36158 & 2.2434 & 3.3823 & 2.186759462 & 3.373556577 \\
\hline 1 & 1.25 & 2.17918 & 3.36965 & 2.2254 & 3.3907 & 2.1822119 & 3.3716756 \\
\hline
\end{tabular} \begin{tabular}{c|c|c|c|c|c|c|c|}
1 & 1.25 & 2.17918 & 3.36965 & 2.2254 & 3.3907 & 2.18221719 & 3.37167556 \\
\hline 1.25 & 1.5 & 2.17918 & 3.36965 & 2.2074 & 3.3977 & 2.176752125 & 3.372181943 \\
\hline
\end{tabular} \begin{tabular}{c|c|c|c|c|c|c|c|c|}
\hline 1.5 & 1.75 & 2.17111 & 3.38579 & 2.1894 & 3.4036 & 2.170350743 & 3.374991672 \\
\hline 1.75 & 2 & 2.159 & 3.38176 & 2.1751 & 3.4084 & 2.163026757 & 3.37979216 \\
\hline & 2.25 & 2.15819 & 3.38983 & 2.1535 & 3.412 & 2.15482032 & 3.38601178 \\
\hline
\end{tabular} \begin{tabular}{|c|c|c|c|c|c|c|c|}
\hline 1.75 & 2 & 2.159 & 3.38176 & 2.1751 & 3.4084 & 2.163026757 & 3.379792164 \\
\hline 2 & 2.25 & 2.15819 & 3.38983 & 2.1535 & 3.412 & 2.154820328 & 3.386051178 \\
\hline
\end{tabular} \begin{tabular}{c|c|c|c|c|c|c|c|}
\hline 2.25 & 2.5 & 2.14689 & 3.38983 & 2.1355 & 3.4146 & 2.145790119 & 3.393092756 \\
\hline
\end{tabular} \begin{tabular}{|c|c|c|c|c|c|c|c|}
\hline 2.5 & 2.75 & 2.13882 & 3.3979 & 2.1176 & 3.4161 & 2.136000000 & 3.400223563 \\
\hline
\end{tabular} \begin{tabular}{|c|c|c|c|c|c|c|c|}
\hline 2.75 & 3 & 2.13075 & 3.40194 & 2.0996 & 3.4166 & 2.12550524 & 3.406863738 \\
\hline 3 & 3.25 & 2.12268 & 3.41001 & 2.0816 & 3.4161 & 2.143436 & 3.412633595 \\
\hline
\end{tabular} \begin{tabular}{|c|c|c|c|c|c|c|c|}
\hline 3 & 3.25 & 2.12268 & 3.41001 & 2.0816 & 3.4161 & 2.11434336 & 3.412633595 \\
\hline 3.25 & 3.5 & 2.10734 & 3.41808 & 2.0636 & 3.4147 & 2.102532065 & 3.417373395 \\
\hline 3.5 & 3.75 & 2.0904 & 3.42373 & 2.0457 & 3.4123 & 2.0907314 & 3.421106618 \\
\hline
\end{tabular} \begin{tabular}{|l|l|l|l|l|l|l|l|}
\hline 3.5 & 3.75 & 2.0904 & 3.42373 & 2.0457 & 3.4123 & 2.090073149 & 3.421106618 \\
\hline 3
\end{tabular} \begin{tabular}{|c|c|c|c|c|c|c|c|}
\hline 3.75 & 4 & 2.0791 & 3.42938 & 2.0277 & 3.4091 & 2.076959378 & 3.423975275 \\
\hline 4 & 4.25 & 2.0678 & 3.42938 & 2.0097 & 3.405 & 2.063181506 & 3.426174076 \\
\hline
\end{tabular} \begin{tabular}{c|c|c|c|c|c|c|c|}
\hline 4.25 & 4.5 & 2.05198 & 3.42147 & 1.9917 & 3.4001 & 2.048733878 & 3.427898365 \\
\hline 4.5 & 4.75 & 2.02986 & 3.43422 & 1.9738 & 3.3945 & 2.03361823 & 3.429309015 \\
\hline
\end{tabular} \begin{tabular}{|l|l|l|l|l|l|l|l|l|}
\hline 4.5 & 4.75 & 2.02986 & 3.43422 & 1.9738 & 3.3945 & 2.033618293 & 3.429309015 \\
\hline
\end{tabular}

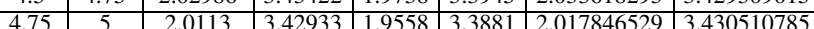
\begin{tabular}{|c|c|c|c|c|c|c|c|}
\hline 5 & 5.25 & 1.99435 & 3.42373 & 1.9378 & 3.381 & 2.001442149 & 3.431538579 \\
\hline
\end{tabular} \begin{tabular}{|c|c|c|c|c|c|c|c|c|}
\hline 5.25 & 5.5 & 1.98144 & 3.43019 & 1.9199 & 3.3732 & 1.984442103 & 3.432346835 \\
\hline 5.5 & 5.75 & 1.96045 & 3.42938 & 1.9019 & 3.3648 & 1.96698343 & 3.43279962 \\
5.75 & 6 & 1.94915 & 3.43019 & 1.8839 & 3.3558 & 1.9487919 & 3.432662761 \\
\hline
\end{tabular} \begin{tabular}{|c|c|c|c|c|c|c|c|c|}
\hline 5.5 & 5.75 & 1.96045 & 3.4293 & 1.9019 & 3.3648 & 1.966898343 & 3.432799628 \\
\hline 5.75 & 6 & 1.9495 & 3.4301 & 1.8839 & 3.358 & 1.988797 & 3.4266261 \\
\hline
\end{tabular} \begin{tabular}{|c|c|c|c|c|c|c|c|c|}
\hline 5.75 & 6 & 1.94915 & 3.4301 & 1.8839 & 3.3558 & 1.948879197 & 3.432662761 \\
\hline 6 & 6.25 & 1.92655 & 3.42938 & 1.8659 & 3.3462 & 1.930469403 & 3.43604735 \\
\hline
\end{tabular} \begin{tabular}{cc|c|c|c|c|c|c|c|}
\hline 6.25 & 6.5 & 1.9096 & 3.43503 & 1.848 & 3.3361 & 1.9911766619 & 3.429221116 \\
\hline 6 & 6.75 & 1.926 & 3.42615 & 1.83 & 3.325 & 1.8287125 & 3.4210302 \\
\hline
\end{tabular} \begin{tabular}{l|l|l|l|l|l|l|l|}
6.5 & 6.75 & 1.89266 & 3.42615 & 1.83 & 3.3255 & 1.892871285 & 3.425103028 \\
\hline
\end{tabular} \begin{tabular}{c|c|c|c|c|c|c|c|}
6.75 & 7 & 1.88055 & 3.42211 & 1.812 & 3.3144 & 1.873867609 & 3.418964553 \\
\hline
\end{tabular} \begin{tabular}{|l|l|l|l|l|l|l|l|l|}
\hline 7 & 7.25 & 1.85876 & 3.41243 & 1.794 & 3.3028 & 1.854798692 & 3.410809045 \\
\hline
\end{tabular} \begin{tabular}{|c|c|c|c|c|c|c|c|c|}
\hline 7.25 & 7.5 & 1.83616 & 3.40678 & 1.7761 & 3.2909 & 1.835648248 & 3.401051244 \\
\hline 7.5 & 7.75 & 1.81356 & 3.4613 & 1.7581 & 3.2286 & 1.81634694 & 3.3045211 \\
\hline
\end{tabular} \begin{tabular}{|c|c|c|c|c|c|c|c|c|}
\hline 7.5 & 7.75 & 1.81356 & 3.40113 & 1.7581 & 3.2786 & 1.81634694 & 3.390475111 \\
\hline 7.75 & 8 & 1.79984 & 3.36158 & 1.740 & 3.266 & 1.768053 & 3.399251 \\
\hline
\end{tabular} \begin{tabular}{|c|c|c|c|c|c|c|c|}
\hline 7.75 & 8 & 1.79984 & 3.36158 & 1.7401 & 3.266 & 1.796810053 & 3.379982051 \\
\hline 8 & 8.25 & 1.77966 & 3.36965 & 1.722 & 3.253 & 1.77698806 & 3.37251308 \\
\hline
\end{tabular} \begin{tabular}{|c|c|c|c|c|c|c|c|}
\hline 8 & 8.25 & 1.77966 & 3.36965 & 1.7222 & 3.2531 & 1.776988061 & 3.370251304 \\
\hline 8.25 & 8.5 & 1.75706 & 3.36723 & 1.7042 & 3.2399 & 1.756898836 & 3.36151085 \\
\hline
\end{tabular}

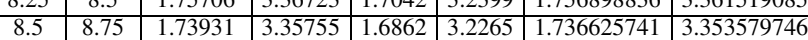
\begin{tabular}{c|c|c|c|c|c|c|c|}
\hline 8.75 & 9 & 1.71913 & 3.34948 & 1.6682 & 3.2129 & 1.716290452 & 3.345948928 \\
\hline & 9.25 & 1.6492 & 3.3463 & 1.6503 & 3.192 & 1.6960866 & 3.3380789 \\
\hline
\end{tabular} \begin{tabular}{|c|c|c|c|c|c|c|c|}
\hline 9 & 9.25 & 1.69492 & 3.34463 & 1.6503 & 3.1992 & 1.69601866 & 3.33806789 \\
\hline
\end{tabular} \begin{tabular}{c|ccccccccc|}
\hline 9.25 & 9.5 & 1.67232 & 3.32768 & 1.6323 & 3.1853 & 1.675911158 & 3.329468411 \\
\hline
\end{tabular} \begin{tabular}{|l|l|l|l|l|l|l|}
\hline 1.65052 & 3.32123 & 1.6143 & 3.1713 & 1.656025072 & 3.319872427 \\
\hline
\end{tabular} \begin{tabular}{ccccccccc|c|}
9.75 & 10 & 1.63035 & 3.30912 & 1.5964 & 3.1573 & 1.636366626 & 3.309226066 \\
\hline
\end{tabular} \begin{tabular}{|c|c|c|c|c|c|c|c|}
\hline 10 & 10.25 & 1.61824 & 3.29701 & 1.5784 & 3.1433 & 1.616895951 & 3.297672933 \\
\hline 10.25 & 10.5 & 1.59887 & 3.28249 & 1.5604 & 3.1292 & 1.597542878 & 3.28547673 \\
\hline
\end{tabular} \begin{tabular}{|l|l|l|l|l|l|l|l|l|}
\hline 10.5 & 10.75 & 1.58192 & 3.26877 & 1.5424 & 3.1153 & 1.578229533 & 3.272915207 \\
\hline
\end{tabular} \begin{tabular}{|llllllllll|l|}
\hline 10.75 & 11 & 1.56174 & 3.25666 & 1.5245 & 3.1013 & 1.558892864 & 3.260178391 \\
\hline 11 & 1.25 & 1.5362 & 3.2526 & 1.5065 & 3.0875 & 1.5350035 & 3.2430329 \\
\hline
\end{tabular} \begin{tabular}{|l|l|l|l|l|l|l|l|}
\hline 11 & 11.25 & 1.53672 & 3.25424 & 1.5065 & 3.0875 & 1.539500355 & 3.247303294 \\
\hline
\end{tabular} \begin{tabular}{|c|c|c|c|c|c|c|c|}
\hline 11.25 & 11.5 & 1.5197 & 3.23729 & 1.4885 & 3.0739 & 1.52005534 & 3.234163114 \\
\hline 11.5 & 1.75 & 1.0121 & 3.2437 & 1.4705 & 3.0604 & 1.500592591 & 3.2205971 \\
\hline
\end{tabular} \begin{tabular}{|c|c|c|c|c|c|c|c|}
\hline 11.75 & 12 & 1.48103 & 3.20823 & 1.4526 & 3.0472 & 1.481167693 & 3.206053827 \\
\hline 12 & 12.25 & 1.46086 & 3.18805 & 1.4346 & 3.0342 & 1.461844345 & 3.190561503 \\
\hline
\end{tabular} \begin{tabular}{|c|c|c|c|c|c|c|c|}
\hline 12 & 12.25 & 1.46086 & 3.18805 & 1.4346 & 3.0342 & 1.461844345 & 3.190561503 \\
\hline 12.25 & 12.5 & 1.44633 & 3.16949 & 1.4166 & 3.0215 & 1.442682842 & 3.173944500 \\
\hline
\end{tabular} \begin{tabular}{|l|l|l|l|l|l|l|l|}
\hline 12.5 & 12.75 & 1.42857 & 3.15981 & 1.3987 & 3.0091 & 1.423731612 & 3.15632401 \\
\hline
\end{tabular} \begin{tabular}{|l|l|l|l|l|l|l|l|}
\hline 12.75 & 13 & 1.40839 & 3.13963 & 1.3807 & 2.9971 & 1.405022572 & 3.13805028 \\
\hline 13 & 13.25 & 1.3805 & 3.11945 & 1.3627 & 2.985 & 1.3855025 & 3.196624 \\
\hline
\end{tabular} \begin{tabular}{|c|c|c|c|c|c|c|c|}
\hline 13 & 13.25 & 1.38015 & 3.11945 & 1.3627 & 2.9854 & 1.386570215 & 3.119669014 \\
\hline 13.25 & 13.5 & 1.36723 & 3.09605 & 1.3447 & 2.9742 & 1.368373589 & 3.101839329 \\
\hline
\end{tabular} \begin{tabular}{|c|c|c|c|c|c|c|c|}
\hline 13.25 & 13.5 & 1.36723 & 3.09605 & 1.3447 & 2.9742 & 1.368373589 & 3.101839329 \\
\hline 13.5 & 13.75 & 1.3519 & 3.08717 & 1.3268 & 2.9634 & 1.350419758 & 3.085223618 \\
\hline
\end{tabular} \begin{tabular}{|l|l|l|l|l|l|l|l|}
\hline 13.75 & 14 & 1.33172 & 3.07103 & 1.3088 & 2.9532 & 1.332686968 & 3.070378841 \\
\hline
\end{tabular} \begin{tabular}{|l|l|l|l|l|l|l|l|}
\hline 14 & 14.25 & 1.31961 & 3.05892 & 1.2908 & 2.9434 & 1315145999 & 3.057675597 \\
\hline
\end{tabular} \begin{tabular}{lllllllll|l|l|}
14.25 & 14.5 & 1.29944 & 3.0565 & 1.2728 & 2.9342 & 1.297758688 & 3.047257546 \\
\hline
\end{tabular} \begin{tabular}{|c|c|c|c|c|c|c|c|}
\hline 14.5 & 14.75 & 1.27926 & 3.03874 & 1.2549 & 2.9256 & 1.280473345 & 3.039037159 \\
\hline
\end{tabular}

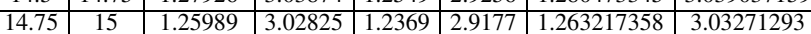
\begin{tabular}{|l|l|l|l|l|l|l|l|}
15 & 15.25 & 1.25101 & 3.03874 & 1.2189 & 2.9104 & 1.245887804 & 3.02779209 \\
\hline
\end{tabular} \begin{tabular}{|c|c|c|c|c|c|c|c|}
\hline 15.25 & 15.5 & 1.22599 & 3.01695 & 1.201 & 2.9038 & 1.228342041 & 3.023611391 \\
\hline 15.5 & 15.75 & 1.090 & 3.013 & 1.83 & 2.897 & 1.2039266 & 3.036557 \\
\hline
\end{tabular} \begin{tabular}{|c|c|c|c|c|c|c|c|}
\hline 15.5 & 15.75 & 1.20904 & 3.0113 & 1.183 & 2.8979 & 1.210392669 & 3.019365567 \\
\hline
\end{tabular} \begin{tabular}{c|c|c|c|c|c|c|c|c|}
\hline 15.75 & 16 & 1.19209 & 3.0113 & 1.165 & 2.8928 & 1.191815554 & 3.014176798 \\
\hline
\end{tabular} \begin{tabular}{|l|l|l|l|l|l|l|l|}
16 & 16.25 & 1.1703 & 3.00646 & 1.147 & 2.8884 & 1.172383691 & 3.007259144 \\
\hline
\end{tabular} \begin{tabular}{|l|l|l|l|l|lll|l|}
\hline 16.25 & 16.5 & 1.15012 & 3.01049 & 1.1291 & 2.885 & 1.151935686 & 2.9982168 \\
\hline 16.5 & 16.75 & 1.1380 & 2.9983 & 1.111 & 2.8824 & 1.13046295 & 2.984289 \\
\hline
\end{tabular} \begin{tabular}{|l|l|l|l|l|l|l|l|l|}
\hline 16.5 & 16.75 & 1.13801 & 2.99839 & 1.1111 & 2.8824 & 1.130462957 & 2.98741289 \\
\hline
\end{tabular} \begin{tabular}{|c|c|c|c|c|c|c|c|}
\hline 16.75 & 17 & 1.10169 & 2.95803 & 1.0931 & 2.8806 & 1.108157717 & 2.976170793 \\
\hline 17 & 17.25 & 1.0859 & 2.950 & 1.075 & 2.879 & 1.0855288 & 2.9652352 \\
\hline
\end{tabular} \begin{tabular}{|c|c|c|c|c|c|c|c|}
\hline 17 & 17.25 & 1.08959 & 2.96207 & 1.0751 & 2.8799 & 1.085352868 & 2.966523582 \\
\hline 17.25 & 17.5 & 1.06215 & 2.96045 & 1.0572 & 2.8801 & 1.062359012 & 2.965532524 \\
\hline
\end{tabular} \begin{tabular}{|l|l|l|l|l|l|l|l|}
\hline 17.25 & 17.5 & 1.06215 & 2.96045 & 1.0572 & 2.8801 & 1.062359012 & 2.960532524 \\
\hline
\end{tabular} \begin{tabular}{|l|l|l|l|l|l|l|l|}
\hline 17.5 & 17.75 & 1.03955 & 2.9661 & 1.0392 & 2.8813 & 1.039305812 & 2.959616637 \\
\hline 17.75 & 18 & 1.0165 & 2.9714 & 1.021 & 2.8836 & 1.0609176 & 2.964213037 \\
\hline
\end{tabular} \begin{tabular}{|l|l|l|l|l|l|l|}
1.01695 & 2.9774 & 1.0212 & 2.8835 & 1.016091761 & 2.964321303 \\
\hline
\end{tabular} \begin{tabular}{|c|c|c|c|c|c|c|c|c|}
18 & 18.25 & 0.988701 & 2.97821 & 1.0033 & 2.8868 & 0.992442652 & 2.974524237 \\
\hline
\end{tabular} \begin{tabular}{|c|c|c|c|c|c|c|c|c|}
\hline 18.25 & 18.5 & 0.971751 & 2.9887 & 0.9853 & 2.8912 & 0.968009009 & 2.989787327 \\
\hline 18.5 & 18.75 & 0.936239 & 3.00646 & 0.9673 & 2.8963 & 0.942446684 & 3.009621486 \\
\hline
\end{tabular} \begin{tabular}{|l|c|c|c|c|c|c|c|c|}
\hline 18.75 & 19 & 0.920097 & 3.02663 & 0.9493 & 2.9035 & 0.915466504 & 3.033605786 \\
\hline
\end{tabular} \begin{tabular}{|c|c|c|c|c|c|c|c|c|}
19 & 19.25 & 0.887006 & 3.05085 & 0.9314 & 2.9114 & 0.886861758 & 3.061400275 \\
\hline
\end{tabular} \begin{tabular}{llllllllll}
19.25 & 19.5 & 0.858757 & 3.0904 & 0.9134 & 2.9206 & 0.856524855 & 3.092706667 \\
\hline
\end{tabular} \begin{tabular}{|l|l|l|l|l|l|l|l|l|}
\hline 19.5 & 19.75 & 0.830508 & 3.11864 & 0.8954 & 2.9311 & 0.824459185 & 3.127214353 \\
\hline
\end{tabular} \begin{tabular}{|l|l|l|l|l|l|l|l|l|}
\hline 19.75 & 20 & 0.79096 & 3.18805 & 0.8774 & 2.9429 & 0.790787012 & 3.164552035 \\
\hline
\end{tabular} \begin{tabular}{|c|c|c|c|c|c|c|c|}
\hline 20 & 20.25 & 0.751412 & 3.19209 & 0.8595 & 2.956 & 0.755751204 & 3.204255063 \\
\hline
\end{tabular} 


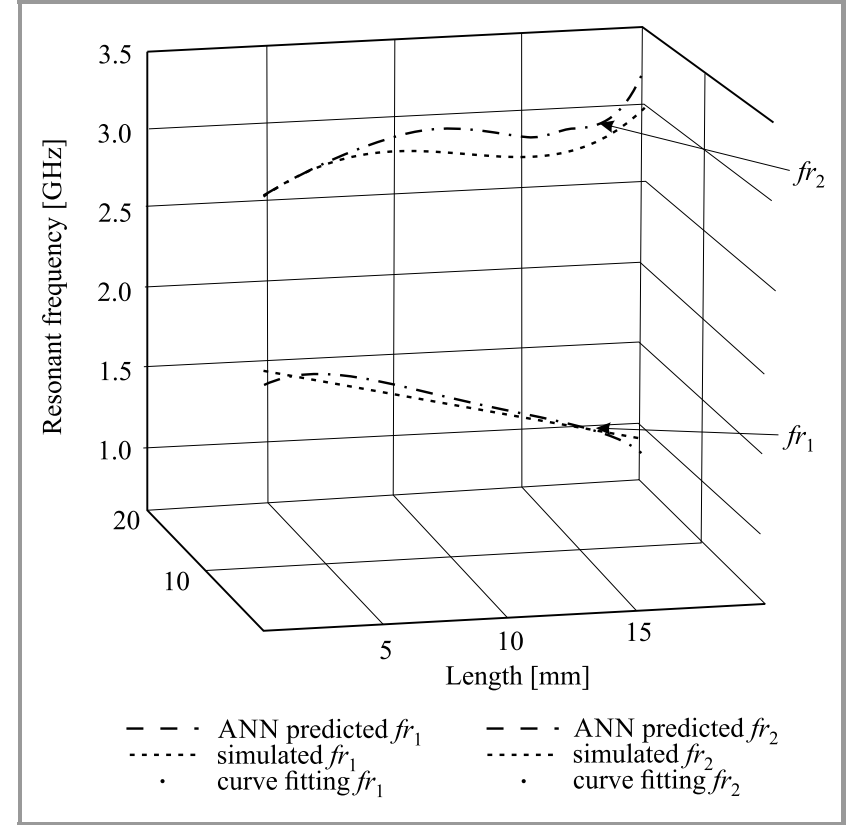

Fig. 10. Notch dimension vs. resonant frequency for $T M_{10}$, and $T M_{02}$ modes of operation.

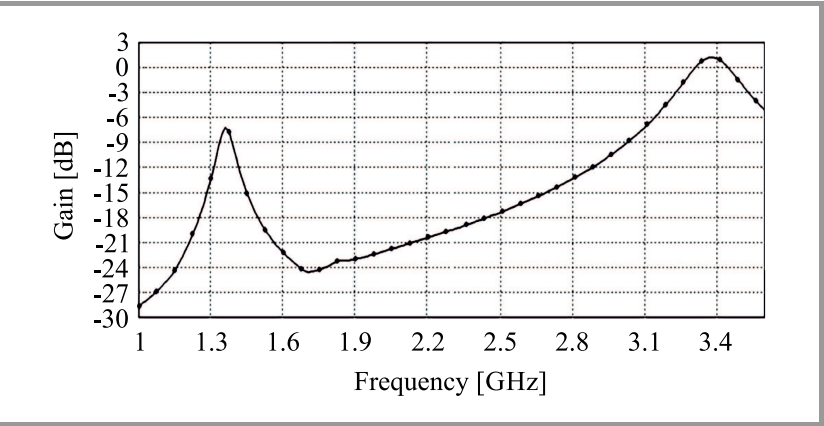

Fig. 11. Maximum gain vs. frequency plot for sample H-shaped RMSA.

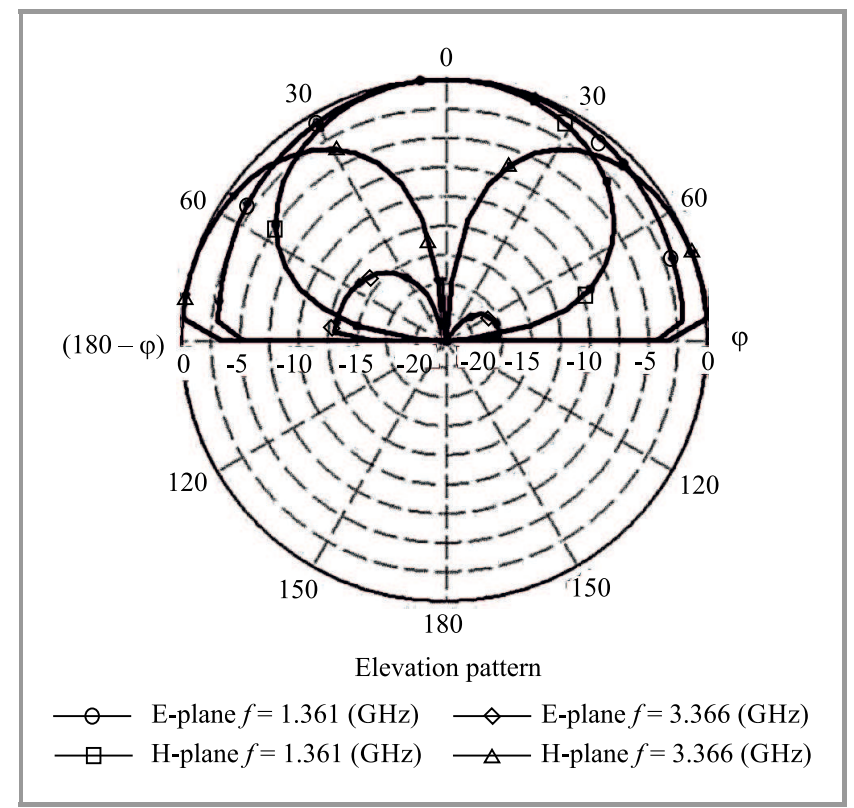

Fig. 12. Radiation pattern for sample H-shaped RMSA. number of samples, in comparison to examples existing literature [40].

\section{Design Specification and Dataset}

Design specifications of the proposed dual-band H-shaped compact RMSA with an infinite ground plane are shown in Fig. 13, and the list of parameters used in the design

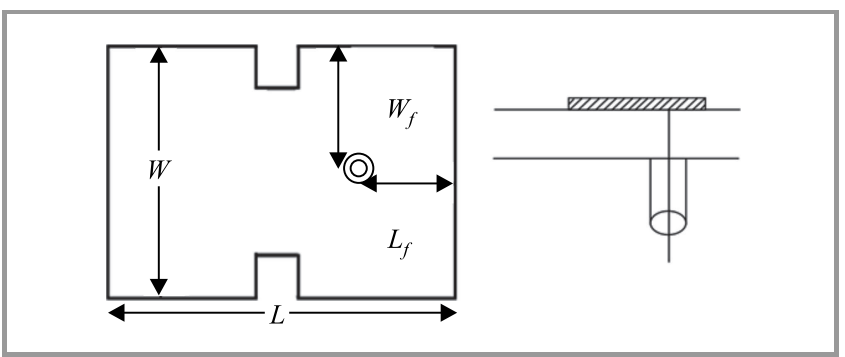

Fig. 13. Top and side view of H-shaped RMSA used for modeling.

is presented in Table 4. Along the radiating edge, two rectangular notches that are equal in dimensions are cut (upper, lower and centered in the middle), starting with the notch dimensions of $0.2 \times 0.5 \mathrm{~mm}$, increased to $20 \times$ $20.25 \mathrm{~mm}$ in fixed steps of $0.25 \mathrm{~mm}$ in $L$ and $W .80$ samples were collected using IE3D simulation software.

Table 4

Specification of H-shaped RMSA

\begin{tabular}{|l|c|}
\hline \multicolumn{1}{|c|}{ Parameter } & Value \\
\hline Operating frequency & $2.33 \mathrm{GHz}$ \\
Patch length & $L=32 \mathrm{~mm}$ \\
Patch width & $W=42 \mathrm{~mm}$ \\
Substrate & FR-4 \\
Substrate thickness & $1.6 \mathrm{~mm}$ \\
Dielectric constant & 4.4 \\
Loss tangent & 0.025 \\
Feed type & Coaxial \\
Notch length & 0.25 to $20 \mathrm{~mm}$ \\
Notch width & 0.5 to $20.25 \mathrm{~mm}$ \\
Feed location $\left(L_{f}, W_{f}\right)$ & $6.575 \mathrm{~mm}, 21 \mathrm{~mm}$ \\
\hline
\end{tabular}

\section{Conclusion}

Machine learning is one of the methods used to design antennas quickly. The proposed model is simple, timeefficient and does not require any complex mathematical calculations. It may easily predict, with high accuracy, the physical or electrical parameters within the range of data provided for training, for a dual-band $(0.75$ to $2.20 \mathrm{GHz}$ and 3.0 to $3.44 \mathrm{GHz}$ ), compact microstrip antenna that may be used in a variety of wireless applications. 


\section{References}

[1] M. Fallahpour and R. Zoughi, "Antenna miniaturization techniques: A review of topology- and material-based methods", IEEE Antenn. and Propag. Maga., vol. 60, no 1, pp. 38-50, 2018

(DOI: 10.1109/MAP.2017.2774138).

[2] P. L. Chi, R. Waterhouse, and T. Itoh, "Antenna miniaturization using slow wave enhancement factor from loaded transmission line models", IEEE Trans. on Antenn. and Propag., vol. 59, no. 1, pp. 48-57, 2011 (DOI: 10.1109/TAP.2010.2090452).

[3] A. Foudazi, H. R. Hassani, and S. M. Ali Nezhad, "Small UWB planar monopole antenna with added GPS/GSM/WLAN bands", IEEE Trans. on Antenn. and Propag., vol. 60, no. 6, pp. 2987-2992, 2012 (DOI: 10.1109/TAP.2012.2194632).

[4] V. K. Sambhe, R. N. Awale, and A. Wagh, "Compact U-shape radiating patch with rectangular ground planar monopole antenna", The J. of Engin., vol. 2015, no. 2, pp. 54-58, 2015 (DOI: 10.1049/joe.2014.0269).

[5] X. Ren, S. Gao, and Y. Yin, "Compact tri-band monopole antenna with hybrid strips for WLAN/WiMAX applications", Microw. and Opt. Technol. Lett., vol. 57, no. 1, pp. 94-99, 2015 (DOI: $10.1002 / \mathrm{mop} .28785)$.

[6] M.-T. Tan, B.-Z. Wang, and Z.-M. Zhang, "Compact tri-band dualpolarized planar monopole antenna with asymmetrical ground plane and loaded stub", Progr. in Electromag. Res. Lett., vol. 58, pp. 89-95 2016 (DOI: 10.2528/PIERL15122004).

[7] Y. Mao, S. Guo, and M. Chen, "Compact dual-band monopole antenna with defected ground plane for Internet of Things", IET Microw., Antenn. Propag., vol. 12, no. 8, pp. 1332-1338, 2018 (DOI: 10.1049/iet-map.2017.0860).

[8] R. O. Ouedraogo, E. J. Rothwell, A. R. Diaz, K. Fuchi, and A. Temme, "Miniaturization of patch antennas using a metamaterialinspired technique", IEEE Trans. on Antenn. and Propag., vol. 60, no. 5, pp. 2175-2182, 2012 (DOI: 10.1109/TAP.2012.2189699).

[9] Y. Dong and T. Itoh, "Metamaterial-based antennas", Proceedings of the IEEE, vol. 100, no. 7, pp. 2271-2285, 2012 (DOI: 10.1109/JPROC.2012.2187631).

[10] A. Bazrkar, A. Gudarzi, and M. Mahzoon, "Miniaturization of rectangular patch antennas partially loaded with $\mu$-negative metamaterials", in Proc. Int. Conf. on Electron., Biomed. Engin. and its Appl. ICEBEA'2012, Dubai, United Arab Emirates, 2012, pp. 289-292 [Online]. Available: http://psrcentre.org/images/extraimages/ 58.\%200112256.pdf

[11] F. Raval, Y. P. Kosta, J. Makwana, and A. V. Patel, "Design implementation of reduced size microstrip patch antenna with metamaterial defected ground plane", in Proc. of Int. Conf. on Commun. and Sig. Process., Melmaruvathur, India, 2013, pp. 186-190 (DOI: 10.1109/iccsp.2013.6577040).

[12] R. A. H. Mahdi and S. M. R. Taha, "Miniaturization of rectangular microstrip patch antenna using topology optimized metamaterial", IEICE Electron. Express, vol. 14, no. 19, 2017 (DOI: 10.1587/elex.14.20170787).

[13] V. G. Ajay and T. Mathew, "Size reduction of microstrip patch antenna through metamaterial approach for WiMAX application", in Proc. of Int. Conf. on Wirel. Commun., Sig. Process. and Network. WiSPNET 2217, Chennai, India, 2017, pp. 379-381 (DOI: 10.1109/WiSPNET.2017.8299782).

[14] J. Chatterjee, A. Mohan, and V. Dixit, "Broadband circularly polarized H-shaped patch antenna using reactive impedance surface", IEEE Antenn. and Wirel. Propag. Lett., vol. 17, no. 4, pp. 625-628, 2018 (DOI: 10.1109/LAWP.2018.2806993).

[15] B. Gupta, S. Nakhate, and M. Shandilya, "A compact wideband microstrip patch antenna with defected ground plane", in Proc. of 4th World Congr. on Inform. and Commun. Technol. WICT 2014, Bandar Hilir, Malaysia, 2014, pp. 51-56 (DOI: 10.1109/WICT.2014.7077301).

[16] M. K. Khandelwal, B. K. Kanaujia, S. Dwari, S. Kumar, and A. Gautam, "Analysis and design of dual band compact stacked microstrip patch antenna with defected ground structure for WLAN/WiMAX applications", AEU - Int. J. of Electron. and Commun., vol. 69, no. 1, pp. 39-47, 2015 (DOI: 10.1016/j.aeue.2014.07.018).

[17] S. Veisee, S. Asadi, and M. Hedayati, "A novel compact defected ground structure and its application in mutual coupling reduction of a microstrip antenna", Turkish J. of Elec. Engin. and Comp. Sci., vol. 24, no. 5, pp. 3664-3670, 2016 (DOI: 10.3906/elk-1404-517).

[18] P. Anitha, A. S. R. Reddy, and M. N. G. Prasad, "Design of a compact $\psi$-shaped micro-strip patch antenna on modified ground planes", in Proc. of Int. Conf. on Recent Adv. in Electron. and Commun. Technol. ICRAECT 2017, Bangalore, India, 2017, pp. 96-99 (DOI: 10.1109/ICRAECT.2017.60).

[19] C. Sharma and V. D. Kumar, "Koch fractalized compact spiral antenna based on Fibonacci sequence", in Proc. of IEEE 5th AsiaPacific Conf. on Antenn. and Propag. APCAP 2016, Kaohsiung, Taiwan, 2016, pp. 157-158 (DOI: 10.1109/APCAP.2016.7843146).

[20] F. Wang, F. Bin, Q. Sun, J. Fan, and H. Ye, "A compact UHF antenna based on complementary fractal technique", IEEE Access, vol. 5, pp. 21118-21125, 2017 (DOI: 10.1109/ACCESS.2017.2756672).

[21] Y. K. Choukiker and J. C. Mudiganti, "Compact hybrid fractal antenna for wideband wireless applications", Int. J. of Microw. and Wirel. Technol., vol. 9, no. 5, pp. 1191-1196, 2017 (DOI: 10.1017/S1759078716001318).

[22] C. Deng, Y. Li, Z. Feng, W. Li, and D. Zhang, "A dual-band circularly-polarized stacked patch antenna with a small frequencyratio", in Proc. IEEE Antenn. and Propag. Soc. Int. Symp. (APSURSI 2013, Orlando, FL, USA, 2013, pp. 942-943 (DOI: 10.1109/APS.2013.6711130).

[23] A. Foroozesh, D. Psychoudakis, J. Homer, and I. Kim, "Compact wideband stacked microstrip patch antenna for a medical application", in 2016 IEEE Int. Symp. on Antenn. and Propag. APSURSI 2016, Fajardo, Puerto Rico, 2016, pp. 283-284 (DOI: 10.1109/APS.2016.7695850).

[24] A. Katyal and A. Basu, "Compact and broadband stacked microstrip patch antenna for target scanning applications", IEEE Antenn. and Wirel. Propag. Lett., vol. 16, pp. 381-384, 2017 (DOI: 10.1109/LAWP.2016.2578723).

[25] Y. Gou, S. Yang, Q. Zhu, and Z. Nie, "A compact dual-polarized double E-shaped patch antenna with high isolation", IEEE Trans. on Antenn. and Propag., vol. 61, no. 8, pp. 4349-4353, 2013 (DOI: 10.1109/TAP.2013.2262664).

[26] C. K. Hsu and S. J. Chung, "Compact antenna with U-shaped openend slot structure for multi-band handset applications", IEEE Trans. on Antenn. and Propag., vol. 62, no. 2, pp. 929-932, 2014 (DOI: 10.1109/TAP.2013.2289996).

[27] M. Tarbouch, A. E. Amri, and H. Terchoune, "Compact CPWfed microstrip octagonal patch antenna with $\mathrm{h}$ slot for WLAN and WiMAX applications", in Proc. of Int. Conf. on Wirel. Technol., Embedd. and Intell. Syst. WITS 2017, Fez, Morocco, 2017 (DOI: 10.1109/WITS.2017.7934638).

[28] A. Roy, S. Bhunia, D. C. Sarkar, and P. P. Sarkar, "Slot loaded compact microstrip patch antenna for dual band operation", Progress in Electromag. Res. C, vol. 73, pp. 145-156, 2017 (DOI: 10.2528/PIERC17020903).

[29] M. T. Islam, M. Samsuzzaman, and M. Z. Mahmud, "A compact slotted patch antenna for breast tumor detection", Microw. and Opt. Technol. Lett., vol. 60, no. 7, pp. 1600-1608, 2018 (DOI: 10.1002/mop.31215).

[30] Y. Li, Q. Liu, Y. Chen, C. Li, Z. Mo, and F. Li, "A compact triple wideband-notched UWB antenna", in Proc. Int. Worksh. on Antenna Technol. iWAT 2018, Nanjing, China, 2018 (DOI: 10.1109/IWAT.2018.8379142).

[31] B. Choudhury, S. Thomas, and R. M. Jha, "Implementation of soft computing optimization techniques in antenna engineering [Antenna applications corner]", IEEE Antenn. and Propag. Mag., vol. 57, no. 6, pp. 122-131, 2015 (DOI: 10.1109/MAP.2015.2439612).

[32] Z. Cai, S. Zeng, Y. Y. Li, and L. Kang, "Automated antenna design using normalized steady state genetic algorithm", in Proc. of NASA/ESA Conf. on Adapt. Hardware and Syst., Noordwijk, Netherlands, 2008 (DOI: 10.1109/AHS.2008.26). 
[33] S. Ledesma, J. Ruiz-Pinales, G. Cerda-Villafaña, and M. G. GarciaHernandez, "A hybrid method to design wire antennas: Design and optimization of antennas using artificial intelligence", IEEE Antenn. and Propag. Mag., vol. 57, no. 4, pp. 23-31, 2015 (DOI: 10.1109/MAP.2015.2453912).

[34] L. Feruglio, "Artificial intelligence for small satellites mission autonomy", Doctoral Dissertation, Politecnico di Torino, 2017 (DOI: 10.6092/polito/porto/2694565).

[35] L. Y. Xiao, W. Shao, F. L. Jin, and B. Z. Wang, "Multiparameter modeling with ANN for antenna design", IEEE Trans. on Antenn. and Propag., vol. 66, no. 7, pp. 3718-3723, 2018 (DOI: 10.1109/TAP.2018.2823775).

[36] A. Landi, P. Piaggi, M. Laurino, and D. Menicucci, "Artificial neural networks for nonlinear regression and classification", in Proc. of 10th In. Conf. on Intell. Syst. Design and Appl., Cairo, Egypt, 2010, pp. 115-120 (DOI: 10.1109/ISDA.2010.5687280).

[37] S. Lek et al., "Application of neural networks to modelling nonlinear relationships in ecology", Ecological Modell., vol. 90, no. 1, 1996, pp. 39-52 (DOI: 10.1016/0304-3800(95)00142-5).

[38] C. G. Christodoulou and A. Patnaik, "Neural networks for antennas", in Morden Antenna Handbook, C. A. Balanis, Ed. Wiley, 2008, pp. 1625-1657 (ISBN: 9780470036341).

[39] M. Kayri, "Predictive abilities of Bayesian regularization and Levenberg-Marquardt algorithms in artificial neural networks: A comparative empirical study on social data", Mathem. and Comput. Appl., vol. 21, no. 2, 2016 (DOI: 10.3390/mca21020020).

[40] V. S. Kushwah and G. S. Tomar, "Design and analysis of microstrip patch antennas using artificial neural network", in Trends in Research on Microstrip Antennas, S. Chattopadhyay, Ed. Rijeka: IntechOpen, 2017 (DOI: 10.5772/intechopen.69522).

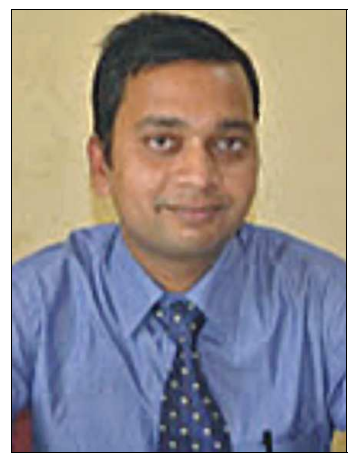

Kanhaiya Sharma received his M.Tech. in Computer Science and Engineering from Jawahar Lal Nehru Technological University (JNTU) Hyderabad, Andhra Pradesh, India in 2011, and currently pursuing a Ph.D. in Computer Science and Engineering from the School of Technology, Pandit Deendayal Petroleum Univer- sity since 2017. He worked as an Assistant Professor at Sri Sarathi Institute of Engineering and Technology, Nuzvid, Krishna District, Andhra Pradesh, India from October 2006 to May 2010, and at Siddhant group of institution, Maharashtra, India from 2010 to 2017. His current research interests include design and development of low-cost solutions for wireless applications, machine learning, wireless communications, microstrip antenna design, filter design, and artificial intelligence.

E-mail: sharmakanhaiya@gmail.com

Department of Computer Science and Engineering

School of Technology

Pandit Deendayal Petroleum University

Gandhinagar, Gujarat 382007, India

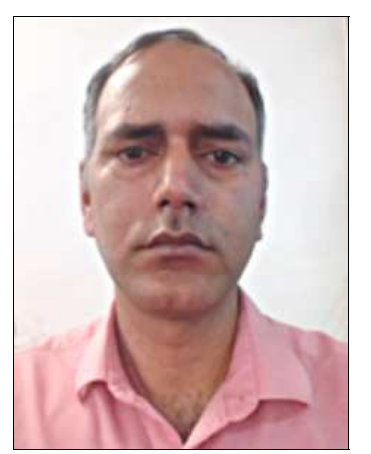

Ganga Prasad Pandey received his B.Tech. in Electronics and Communication Engineering from KNIT, Sultanpur, UP, an M.E. from Delhi College of Engineering (now Delhi Technological University), Delhi and a Ph.D. from Uttarakhand Technical University, Dehradun. He worked as an Assistant Professor at the Maharaja Agasen Institute of Technology, Delhi, from 2002 to 2016. He joined Pandit Deendayal Petroleum University, Gandhinagar in 2016, working at the ICT Department. His current research interests include machine learning in antennas, energy harvesting, ME-dipoles, active, reconfigurable, frequency-agile microstrip antennas, as well as microwave/millimeter wave integrated circuits and devices.

E-mail: Gangaprasad.Pandey @ sot.pdpu.ac.in

Department of Information and Communication

Technology

School of Technology

Pandit Deendayal Petroleum University

Gandhinagar, Gujarat 382007, India 